FACTA UNIVERSITATIS

Series: Mechanical Engineering Vol. 17, Nº 2, 2019, pp. 125 - 139

https://doi.org/10.22190/FUME190301031N

Original scientific paper

\title{
ON THE DISCRETIZATION OF A BISTABLE CANTILEVER BEAM WITH APPLICATION TO ENERGY HARVESTING
}

\author{
Max-Uwe Noll, Lukas Lentz, Utz von Wagner
}

Chair of Mechatronics and Machine Dynamics, Technische Universität Berlin

\begin{abstract}
A typical setup for energy harvesting is that of a cantilever beam with piezoceramics excited by ambient base vibrations. In order to get higher energy output for a wide range of excitation frequencies, often a nonlinearity is introduced by intention in that way, that two magnets are fixed close to the free tip of the beam. Depending on strength and position of the magnets, this can either result in a monobi- or tristable system. In our study, we focus on a bistable system. Such systems have been investigated thoroughly in literature while in almost all cases the beam has been discretized by a single shape function, in general the first eigenshape of the linear beam with undeflected stable equilibrium position.

There can be some doubts about the suitability of a discretization by a single shape function mainly due to two reasons. First: In case of stochastic broadband excitations a discretization, taking into consideration just the first vibration shape seems not to be reasonable. Second: as the undeflected position of the considered system is unstable and the system significantly nonlinear, the question arises, if using just one eigenshape of the linear beam is a suitable approximation of the operation shapes during excited oscillations even in the case of harmonic excitation. Are there other, e.g. amplitude dependent, possibilities and/or should multiple ansatz functions be considered instead?

In this paper, we focus mainly on the second point. Therefore, a bistable cantilever beam with harmonic base excitation is considered and experimental investigations of operation shapes are performed using a high-speed camera. The observed operation shapes are expanded in a similar way as it is done in a theoretical analysis by a corresponding mixed Ritz ansatz. The results show the existence of distinct superharmonics (as one can expect for a nonlinear system) but additionally the necessity to use more than one shape function in the discretization, covering also the amplitude dependence of the observed operation shapes.
\end{abstract}

Key Words: Bistable Beam, Energy Harvesting, Discretization, Vibration Shape Analysis via High Speed Camera

Received March 01, 2019 / Accepted June 20, 2019

Corresponding author: Max-Uwe Noll

Chair of Mechatronics and Machine Dynamics, Technische Universität Berlin, Einsteinufer 5, 10587 Berlin

E-mail: max-uwe.noll@tu-berlin.de 


\section{INTRODUCTION}

Harvesting of energy from ambient vibrations has attracted much interest and corresponding research in the past decades. A common method for transferring the mechanical energy into electric one is the usage of piezoceramics fixed on corresponding vibration structures. A survey of such systems gives, for example, the paper [1]. The classical setup in that case is a cantilever beam with piezoceramics bonded close to the clamping excited by ambient base vibrations. These systems perform well when they are designed as a linear resonator with its eigenfrequency tuned to the frequency of the excitation. A higher energy output, especially for broadband or stochastic excitations, can be realized in such systems, if nonlinearities are introduced by intention resulting in a broadband characteristic for large responses of excited vibrations compared to sharp resonance peaks of the linear system [2]. A common setup is to fix two magnets symmetrically close to the free end of the beam, as shown in Fig. 1.

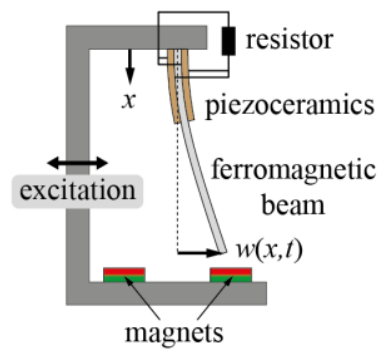

Fig. 1 Vibrational energy harvesting system [3]

Depending on strength and position of the magnets, this can either result in a mono-, bior tristable (e.g. $[4,5,6]$ respectively) system. In this paper we investigate the bistable configuration and focus on the occurring vibration shapes during non-chaotic operation. In that case, there are two main types of solutions, namely so-called intrawell solutions around one of the two stable equilibrium positions and so-called interwell solution with large displacements covering both stable equilibrium positions. For the modeling of the system, knowledge is necessary for the discretization of the beam with respect to its longitudinal coordinate $x$, introducing a mixed (dependence on both $x$ and time $t$ ) Ritz ansatz

$$
w(x, t)=\sum_{i=1}^{n} W_{i}(x) p_{i}(t)
$$

into the partial differential equation describing the continuum vibrations of the beam. Herein $w(x, t)$ is the lateral displacement of the beam relatively to the moving frame and $n$ the chosen ansatz order. In this ansatz shape functions $W_{i}(x)$ shall be given (they must fulfill all the boundary conditions) while functions $p_{i}(t)$ can be calculated from the thereby discretized model equations e.g. by Harmonic Balance. This let arise the question of suitable shape functions $W_{i}(x)$ and ansatz orders $n$.

For the sake of simplicity and in order to concentrate on the question of how to discretize a bistable beam, the piezoceramics are neglected in the following. Only the 
setup of the cantilever beam with magnets and base excitation is considered. The classical paper describing a corresponding modeling is that of Moon [7] discretizing the beam with the first eigenshape of the linear Euler-Bernoulli beam and modeling the magnetic forces by a third order polynomial. This discretization results in a Duffing-oscillator with negative linear and positive cubic restoring term. Most publications follow this model when adding the piezoceramics for the energy harvesting system, e.g. [8] where it was shown, that the nonlinear system performs better than its linear counterpart for a non-resonant excitation and [9] where the system was analyzed for the case of a stochastic excitation. Publications using more than one ansatz function are very rare. In [10] multiple ansatz functions are applied for a buckled beam system, nevertheless ending finally up again with a onedegree of freedom model.

There can be some doubts about the suitability of this discretization by a single shape function mainly due to two reasons. First: In the case of stochastic broadband excitation a discretization, taking into consideration just the first vibration shape, seems not to be reasonable. Therefore, the authors have in prior investigations $[11,12]$ added a second ansatz function in that way, that not just the first, but also the second eigenfunction of the linear beam is considered in the Ritz ansatz. Second: as the undeflected position of the considered system is unstable and the system significantly nonlinear, the question arises if using just the first eigenshape of the linear beam is a suitable approximation of the operation shapes during excited oscillations even in the case of harmonic excitation. Are there other, e.g. amplitude dependent, possibilities and should multiple ansatz functions be considered instead? In the work [13] and [14] this topic was already addressed, and it is shown how the usage of linear shape functions leads to erroneous results. Furthermore, a purely theoretical method to compute nonlinear, i.e. amplitude dependent shape functions, is presented. Later the concept of nonlinear normal-modes was transferred to the analysis of discrete nonlinear systems, see e.g. the review [15] and the references therein.

On the other hand in [16] it is shown, that for a bistable system, in that case a buckled beam, the two first modes coexist during the snapping process. In [17] exact solutions of postbuckling configurations of beams are calculated, but also the interaction between vibration modes is shown. Nevertheless, as already mentioned, it is state of the art in considering energy harvesting systems to use just one mode shape for discretization. An example for this is [18], where both the buckled beam as in the two papers mentioned before as well as the bistable cantilever beam, that is considered in the actual paper, is discretized by a single degree of freedom.

In this paper, the questions, if the mixed Ritz ansatz, Eq. (1), gives a suitable modeling as well as how many and which ansatz functions should be used, is discussed in the case of harmonic base excitation by analyzing experimental results. Therefore, operation shapes have been captured by a high-speed camera. The observed operation shapes are expanded into their frequency content and then the vibration shape corresponding to each frequency is analyzed.

\section{EXPERIMENTAL SETUP}

The chosen setup for the experimental investigations is shown in Fig. 2. The steel cantilever beam is placed in an aluminum frame excited by a shaker and the bistability is realized by two magnets placed approximately symmetrical to the undeflected position of 
the beam, therefore becoming unstable. The beam's static deflection, when there is no base excitation, as well as the dynamic displacement of the beam tip are measured using a laser triangulation sensor which is attached to the moving frame, hence directly providing the relative displacement of the beam tip from its undeflected position without the superposed movement of the supporting frame. The base excitation of the system is captured by a (nonmoving) laser vibrometer. Laptop I is used to process the data delivered by these two measurement devices with the software package vAnalyzer.

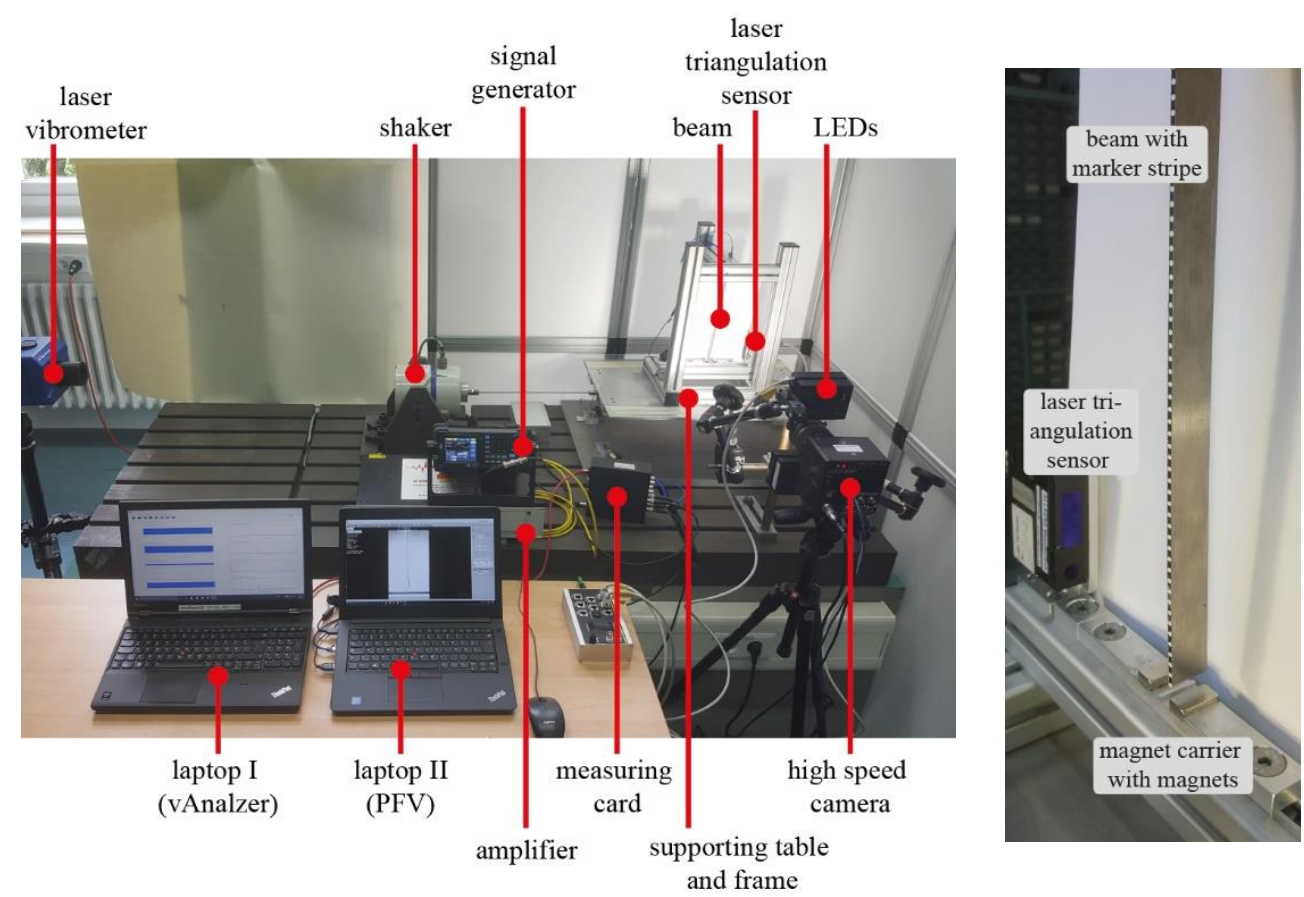

Fig. 2 Experimental setup with bistable cantilever beam excited by a shaker (left) and beam with magnets in detail (right) [19]

Laptop II is utilized to control the high-speed camera Photron Fastcam Mini AX100 by the software package Phontron FASTCAM Viewer for high speed digital imaging. The dimensions of the cantilever beam together with its first natural frequency (without magnets) are given in Table 1.

Table 1 Properties of the cantilever beam

\begin{tabular}{lc}
\hline Property & Value \\
\hline Beam length & $250 \mathrm{~mm}$ \\
Beam width & $20 \mathrm{~mm}$ \\
Beam thickness & $1 \mathrm{~mm}$ \\
1st eigenfrequency & $13.1 \mathrm{~Hz}$ \\
\hline
\end{tabular}


The magnets are intended to be placed symmetrically with respect to the undeflected position of the beam in order to realize an approximately equal distance of the two equilibrium positions from the undeflected beam position. The magnets are glued to a magnet carrier, also made of aluminum, which ensures a constant and reproducible distance between the magnets of approximately $14 \mathrm{~mm}$. The exact placement of the magnet carrier with respect to the beam is however limited by the manual adjustment of the carrier with finite accuracy and reproducibility. The resulting distances of two realized experiments together with the frequencies of small free vibrations around the two stable equilibrium positions (intrawell solutions) are given in Table 2.

Table 2 Properties of bistable beam configuration (magnet distance approx. $14 \mathrm{~mm}$ )

\begin{tabular}{lcc}
\hline & left & right \\
\hline Setup I (static experiment in Fig. 6) & & \\
Equilibrium position & $-6.91 \mathrm{~mm}$ & $7.13 \mathrm{~mm}$ \\
Frequency & $15.1 \mathrm{~Hz}$ & $14.5 \mathrm{~Hz}$ \\
& & \\
Setup II (dynamic experiments) & & \\
Equilibrium position & $-7.01 \mathrm{~mm}$ & $7.40 \mathrm{~mm}$ \\
Frequency & $16.0 \mathrm{~Hz}$ & $16.0 \mathrm{~Hz}$ \\
\hline
\end{tabular}

A paper stripe with geometry given in Fig. 3 is attached to the front side of the beam. The beam itself has a thickness of one millimeter, but for a higher detection rate of the markers their size is chosen to be two millimeters in diameter on an all-black background of the three millimeters wide paper stripe, which therefore exceeds the thickness of the beam.

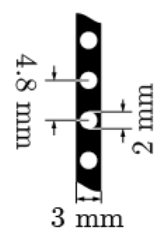

Fig. 3 Geometry of marker stripe

The camera was positioned manually in front of the beam setup with three goals regarding its arrangement. First: the camera was orientated orthogonal to the beam's plane of motion. Second: the camera was placed as far as possible from the beam to reduce errors due to perspective influences. Third: the camera was placed as close as needed so that the beam took up almost the full height of the pictures in order to use the full resolution to capture each marker.

The camera is taking monochrome black and white videos with a frame rate of 4000 frames per second and each frame has a resolution of $384 \times 944$ pixels. Each video has a duration of at least one second. To further reduce the amount of data and processing time only every second frame was considered for further evaluation. The authors are aware that taking a picture is a sampling like process of the time continuous movement of the markers. That means that not only the sampling rate needs to be at least two times the highest frequency that is expected to be occurring in the measured signal (Nyquist criteria), but 
also needs to be filtered for even higher frequencies to prevent aliasing effects. Since there is no possible way to filter these analog signals, we have checked the results from the digital image analysis to the frequencies determined by the laser triangulation sensor, for which the signal has been aliasing filtered properly before sampling. Both results are in good accordance with each other.

The very first frame of each video is replaced by a frame showing the static, undeflected beam without magnets (Fig. 4 (left)), which was taken before the magnets were applied. This first frame is taken as the reference to determine the relative displacement of each marker on each frame from its position when the beam is undeflected. The software GOM Correlate is used to analyze each frame of the video and to detect the relative distance from its position on the reference frame. The calibration of the distance measurement from the frames is done using the known distance of the markers on the maker strip, which results in a resolution of roughly $3.5 \mathrm{pixel} / \mathrm{mm}$.
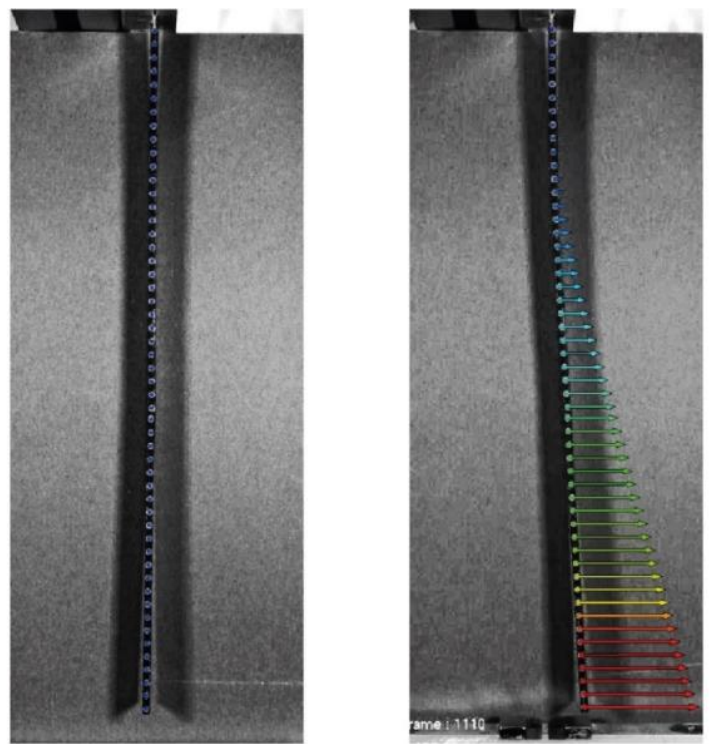

Fig. 4 Reference frame of undeflected beam without magnets (left) and marker detection and evaluation of marker displacement with GOM Correlate (right)

Further it is necessary to eliminate the relative movement of the supporting frame to the nonmoving camera in order to find the relative displacement of each marker. This is done by subtracting the current displacement of the marker that is the nearest to the beam clamping from all other marker displacements of that current frame.

\section{RESULTS OF EXPERIMENTAL INVESTIGATIONS AND THEIR ANALYSIS}

The aim of the following investigations is to decide if the observed operational vibration shapes of the harmonically excited bistable beam can be expanded in the eigenshapes of the Euler-Bernoulli beam as well as how many ansatz functions are required according to the 
mixed Ritz ansatz (Eq. (1)). Fig. 5 shows the first two eigenshapes $\phi_{1}, \phi_{2}$ of the beam together with the static bending line caused by a constant lateral tip force.

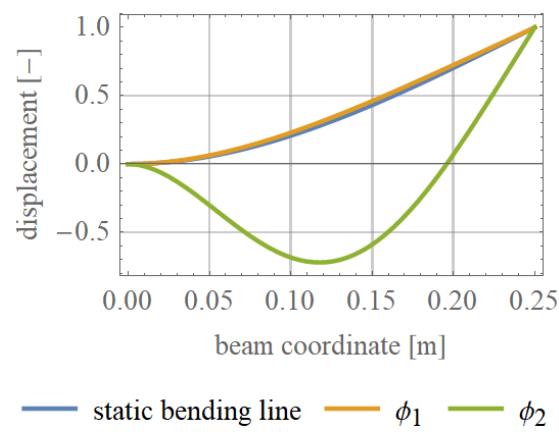

Fig. 5 Beam eigenshapes and static bending line according to the linear Euler-Bernoulli beam theory

In Fig. 6 the measured static bending line resulting in the "right" stable equilibrium (positive displacement according to Table 2) resulting from the magnets from the setup I is displayed. This measured bending line shows a high agreement with the theoretical static bending line.

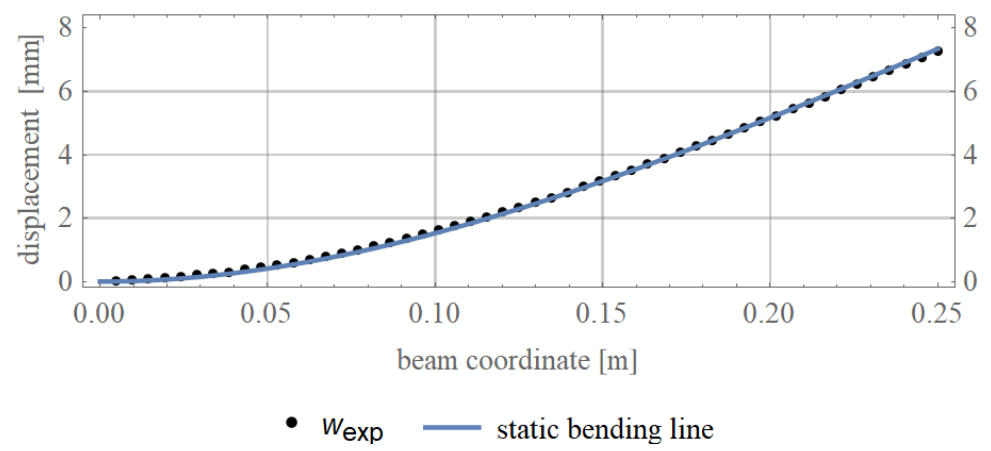

Fig. 6 Static bending of the beam in "right" equilibrium position (Table 2, setup I)

Now the operation shapes are measured with a high-speed camera. Fig. 7 shows typical examples of the two main types of solutions, namely the intrawell solution (left) and the interwell solution (right). The phase diagram of the last marker at the tip of the beam is shown, where the velocity has been determined by the time derivative of the displacement after filtering the signal by a Butterworth low pass filter.

Both solution types do not show any period multiplication in these tests, which restricts in the stationary case the occurring response frequencies to the excitation frequency and corresponding superharmonics while subharmonics do not occur. 

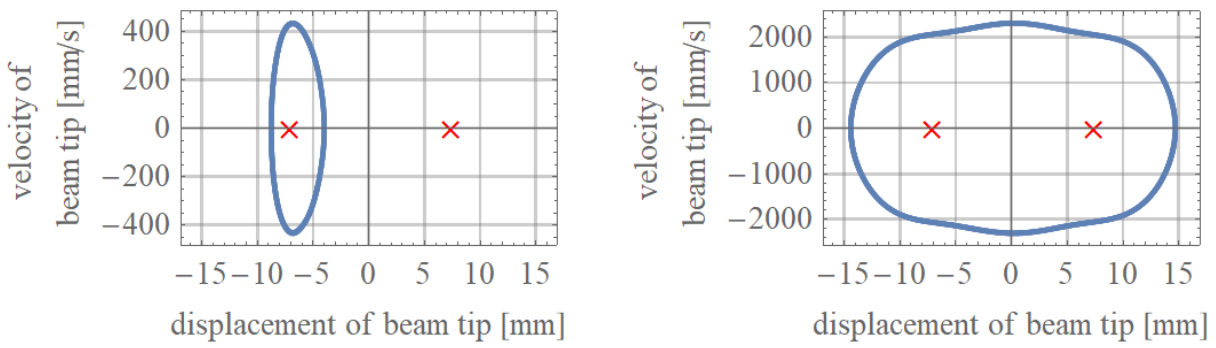

Fig. 7 Phase diagram of beam tip: intrawell solution (left) and interwell solution (right) (setup II)

For distinct points $\hat{x}_{j}$ time series for the displacement can be derived. A corresponding result is shown in Fig. 8 in the case of harmonic excitation with $f_{0}=14 \mathrm{~Hz}$.
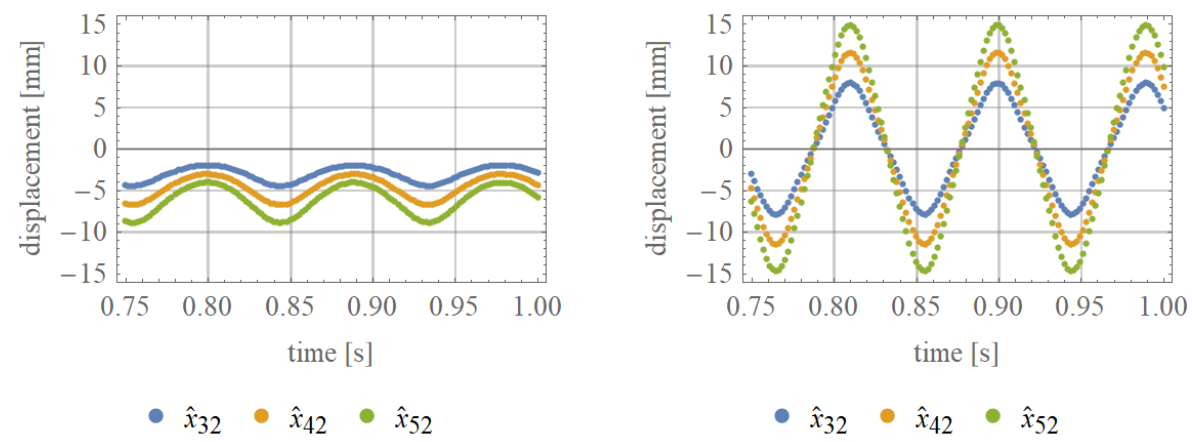

Fig. 8 Time series of three points $x_{j}, j=32,42,52$ of the beam in case of an intrawell (left) and interwell solution (right) resulting from harmonic excitation with $f_{0}=14 \mathrm{~Hz}$ (setup II)

Again in Fig. 8 on the left an intrawell solution is shown while an interwell solution is displayed on the right. An FFT (Fast Fourier Transformation) analysis for each of the time series $\hat{x}_{j}\left(t_{i}\right)$ is performed. A corresponding result is shown in Fig. 9.
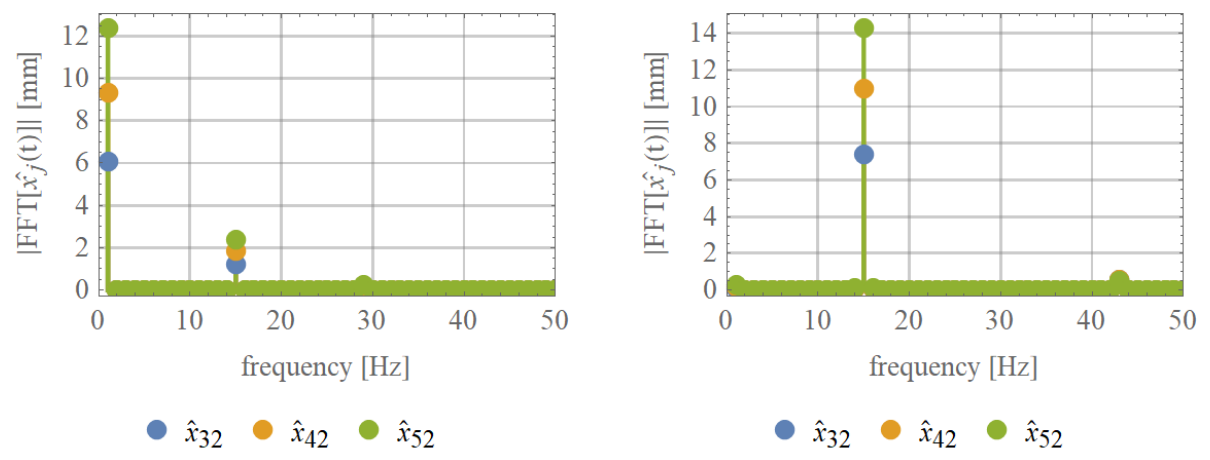

Fig. 9 FFT analysis of the time series in Fig. 8 (setup II) 
According to the nonlinear character of the system, distinct superharmonics of the excitation frequency in the response can be expected. Due to the symmetric characteristic of the interwell solutions, odd superharmonics are expected while even and odd superharmonics can be expected in the intrawell case due to the asymmetric restoring characteristic around the stable equilibrium positions originating from the magnets. This is confirmed by the experimental results. Subharmonics do not occur, as there are no period multiplications.

Having considered these initial results, we will first reconsider the mixed Ritz ansatz (Eq. (1)) and the corresponding theoretical analysis. Using e.g. Harmonic Balance as solution method for the discretized system equations and considering an excitation being proportional to $\cos \Omega t$ with $\Omega=2 \pi f_{0}$ where $\Omega$ is the circular excitation frequency, one can expect, that time function $p_{i}(t)$ in the mixed Ritz ansatz can be expanded as follows

$$
p_{i}(t)=\sum_{k=0}^{m} A_{i k} \cos \left(k \Omega t+\varphi_{i k}\right)
$$

where $k=0,1,2,3, \ldots$ in the case of the intrawell solution and $k=1,3,5,7, \ldots$ in the interwell case. The intrawell case contains both a constant solution part $(k=0)$ due to the deflected stable equilibrium position as well as even superharmonics due to the non-symmetric magnet force characteristic. On the other hand, the interwell solution has in theory a zero mean value and is symmetric, which limits $k$ to odd numbers. These considerations are almost fully confirmed by the results shown in Fig. 8 and 9. Only a small constant part of the interwell solution is possibly due to the non-perfect symmetry of the magnets (Table 2). Inserting the expansion (2) in the mixed Ritz ansatz results after sorting in

$$
w(x, t)=\sum_{i=1}^{n} W_{i}(x) \sum_{k=0}^{m} A_{i k} \cos \left(k \Omega t+\varphi_{i k}\right),
$$

As already mentioned in the introduction, we are interested in the question which and how many shape functions $W_{i}(x)$ are necessary for a good representation of intra- and interwell solutions in our setup. Therefore, we will now do the same expansion with the experimental results. For the $j$-th distinct point $\hat{x}_{j}$ (position on the beam) the time series of its movement is expanded by a Fourier expansion

$$
w\left(\hat{x}_{j}, t\right)=\sum_{k=0}^{m} \tilde{a}_{j k} \cos \left(k \Omega t+\tilde{\varphi}_{j k}\right)
$$

with coefficients $\tilde{a}_{j k}$ and a phase shift $\tilde{\varphi}_{j k}$. While $\varphi_{i k}$ in Eq. (2) describe the phase shift compared to the excitation, $\tilde{\varphi}_{j k}$ depend on the time sequence of the video to be analyzed, and are triggered by the starting of the measurement. As we are interested purely in vibration shapes this is not a restriction.

For each considered multiple $k$ of excitation circular frequency $\Omega$, from $\tilde{a}_{j k}$ a shape $\hat{w}_{k}(x)$ shall be formed, which is then expanded in considered shape functions $W_{i}(x)$. To do so, the following circumstances must be taken into consideration. $\tilde{a}_{j k}$ are taken from the absolute values of the Fourier expansion (which is performed in a complex notation), therefore they have positive values only. This means that in the cases where the beam vibration has vibration nodes it is to be considered that there is a phase shift of $\pi$ between 
$\tilde{\varphi}_{j k}$, even for one fixed mode $k$. Therefore, applicability of Eq. (4) requires, that for each $k$ all $\tilde{\varphi}_{j k}$ are constant for all $j$ with the exception that a jump with size $\pi$ is possible in the case of nodes. If this is the case, subscript $j$ can be neglected in the following and the final phase shift $\hat{\varphi}_{k}$ is described by

$$
\hat{\varphi}_{k}=\tilde{\varphi}_{1 k}
$$

while $\tilde{a}_{j k}$ are replaced by $\hat{a}_{j k}$ following the scheme

$$
\hat{a}_{j k}=\left\{\begin{array}{r}
\tilde{a}_{j k} \text { if }\left|\tilde{\varphi}_{j k}-\tilde{\varphi}_{1 k}\right|=0 \\
-\tilde{a}_{j k} \text { if }\left|\tilde{\varphi}_{j k}-\tilde{\varphi}_{1 k}\right|=\pi .
\end{array}\right.
$$

From $\hat{a}_{j k}$ the shape $\hat{w}_{k}(x)$ is formed, which is expanded as follows

$$
\hat{w}_{k}(x)=\sum_{i=1}^{n} \hat{A}_{i k} W_{i}(x)
$$

with coefficients $\hat{A}_{i k}$. In general, displacement $w(x, t)$ from the experiments is therefore given by

$$
w(x, t)=\sum_{k=0}^{m} \hat{w}_{k}(x) \cos \left(k \Omega t+\hat{\varphi}_{k}\right)
$$

Inserting Eq. (7) in (8) results in

$$
w(x, t)=\sum_{k=0}^{m} \sum_{i=1}^{n} \hat{A}_{i k} W_{i}(x) \cos \left(k \Omega t+\hat{\varphi}_{k}\right)
$$

and after sorting in

$$
w(x, t)=\sum_{i=1}^{n} W_{i}(x) \sum_{k=0}^{m} \hat{A}_{i k} \cos \left(k \Omega t+\hat{\varphi}_{k}\right)
$$

which is almost equal to the theoretical result (3). The only differences are that subscript $i$ is missing in $\hat{\varphi}_{k}$, i.e. if the expansion is possible as described, the phase shift for the $k$-th harmonic does not depend on the number $i$ of the mode $W_{i}(x)$, and reference times for the phase shifts may differ as described above.

In the following, the steps (4)-(7) are performed with the experimental results. Fig. 10 shows a more detailed analysis of the frequency contents of the intrawell solution (left) and interwell solution (right) of the beam tip with a logarithmic scale. Constant parts (zero frequency) are not considered in the following as they are due to the static bending line, which is geometric almost similar to the first eigenshape $\phi_{1}$ (Fig. 4) and therefore anyway covered by the following expansion in $\phi_{1}$ and $\phi_{2}$. In the case of the intrawell solution we limit our result in accordance with Fig. 10 (left) to $k=1,2$ and 3, while we will take in the case of the interwell solution $k=1,3,5$ and 7 into consideration. 

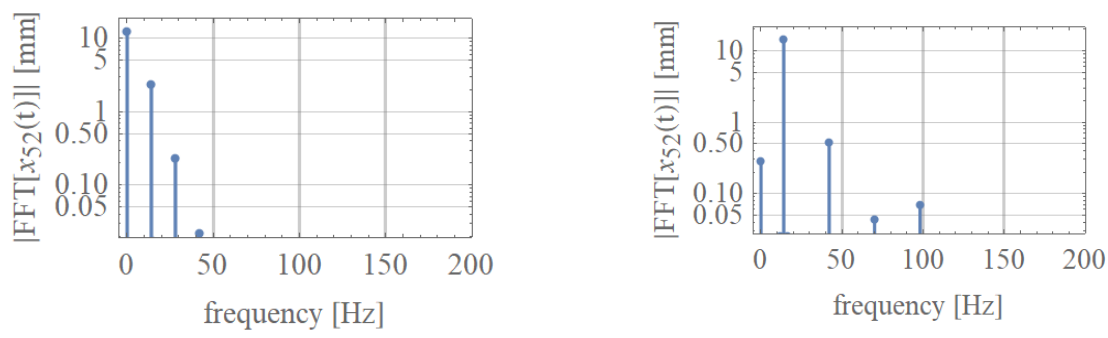

Fig. 10 FFT of beam tip displacement of intrawell (left) and interwell (right) oscillation (setup II)

Fig. 11 shows $\hat{a}_{j 1}$ for the intrawell and interwell solution respectively, forming the corresponding shapes $\hat{w}_{1}(x)$. In the following these shapes shall be expanded according to Eq. (7), where coefficients $\hat{A}_{i k}$ are found from the experimental data using a least square approach. As shape functions $W_{i}(x)$ we take the first two eigenfunctions $\phi_{1}, \phi_{2}$ of the beam as sketched in Fig. 5, i.e. we limit $n$ by 2, which means $i=1,2$. The corresponding expansion shows, that shapes $\hat{w}_{1}(x)$ are almost identical to $\hat{A}_{11} \phi_{1}$, and $\hat{A}_{21} \approx 0$ (red lines in Fig. 11 (top)).

Fig. 11 (bottom) shows the corresponding phases $\tilde{\varphi}_{j 1}$, which should be equal, or only have a jump of $\pi$ at a vibration node, for all $j$ in order to allow the expansion (4). In fact, it can be seen that there are only small deviations occurring mainly close to the clamping, i.e. at points with only small displacements.

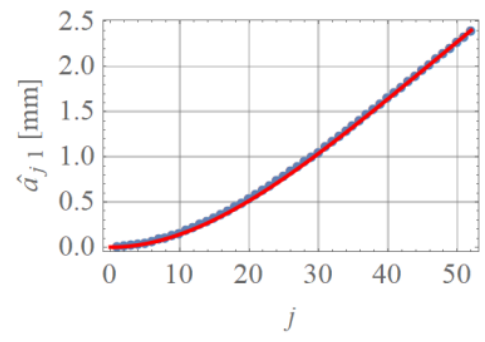

- experimental $-\hat{w}_{1}=\hat{A}_{11} \phi_{1}$

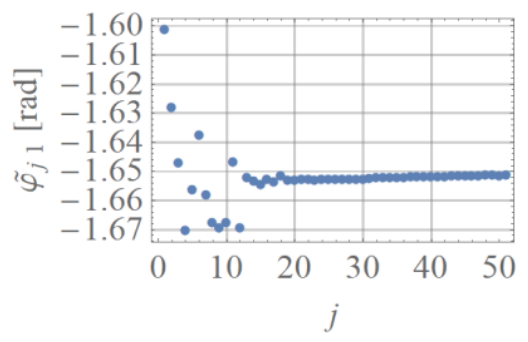

- experimental

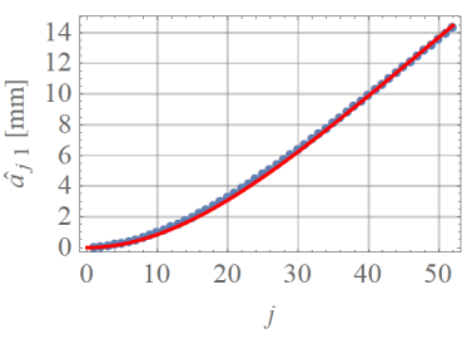

- experimental $-\hat{w}_{1}=\hat{A}_{11} \phi_{1}$

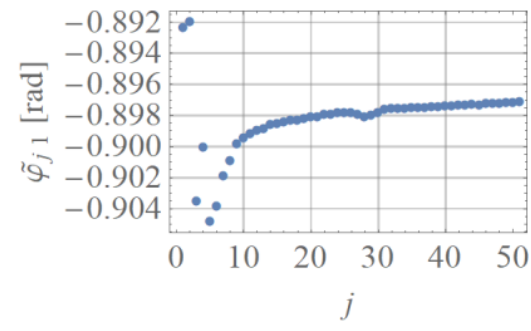

- experimental

Fig. 11 Vibration shape $\hat{a}_{j 1}$ of intrawell (top left) and interwell (top right) for frequency $f=f_{0}$ and corresponding phases $\tilde{\varphi}_{j}$ beneath (setup II) 
For the intrawell solution Fig. 12 shows the $\hat{a}_{j 2}$ and $\hat{a}_{j 3}$ forming the shapes $\hat{w}_{2}(x)$ and $\hat{w}_{3}(x)$ corresponding to twice and three times excitation frequency $2 f_{0}$ and $3 f_{0}$.
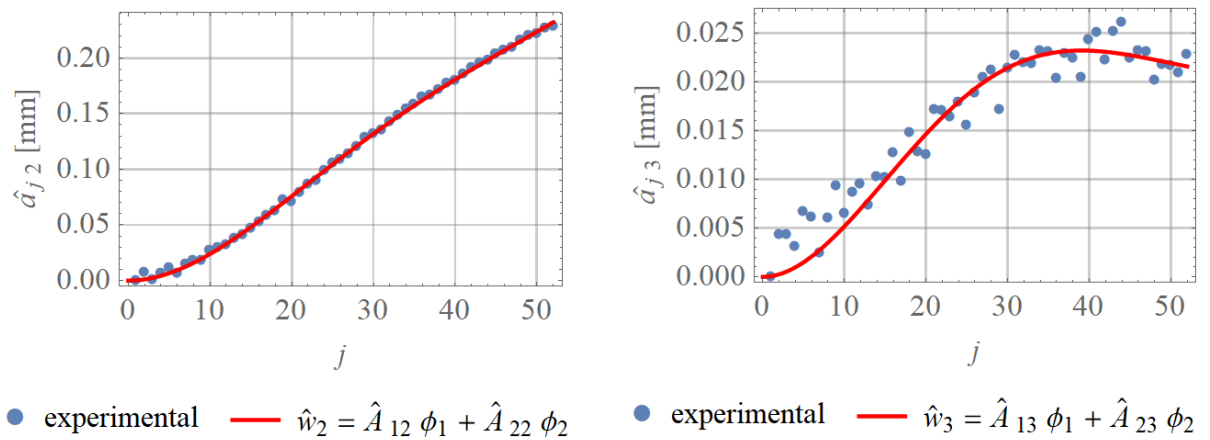

Fig. 12 Vibration shape of intrawell oscillation for frequency $f=2 f_{0}$ (left) and $f=3 f_{0}$ (right) (setup II)

While $\hat{w}_{2}(x)$ gives a somewhat smooth curve there are several small deviations in $\hat{w}_{3}(x)$ but the shapes of both functions can be expanded with the chosen ansatz functions $\phi_{1}$ and $\phi_{2}$.

Fig. 13 shows this step in the case of the interwell solutions for shapes $\hat{w}_{3}(x), \hat{w}_{5}(x)$ and $\hat{w}_{7}(x)$. All shapes can be expanded in good approximation by $\phi_{1}$ and $\phi_{2}$.

- experimental

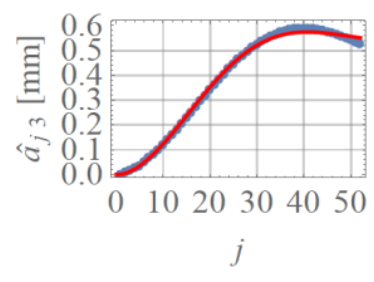

$-\hat{w}_{3}=\hat{A}_{13} \phi_{1}+\hat{A}_{23} \phi_{2}$
- experimental

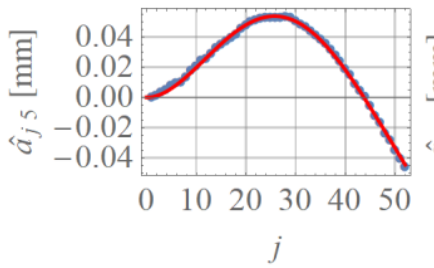

$-\hat{w}_{5}=\hat{A}_{15} \phi_{1}+\hat{A}_{25} \phi_{2}$
- experimental

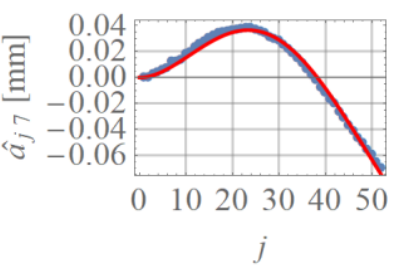

$-\hat{w}_{7}=\hat{A}_{17} \phi_{1}+\hat{A}_{27} \phi_{2}$

Fig. 13 Vibration shape for interwell oscillation for frequency $f=3 f_{0}$ (left), $f=5 f_{0}$ (middle) and $f=7 f_{0}$ (right) (setup II)

From these results it can be concluded that expansions (3) and (10), respectively, can give a good approximation of the experimentally observed shapes and that the beam eigenshapes are suitable functions for the expansion, which is in agreement to most used models in literature (cf. section 1).

On the other hand, the shapes corresponding to the superharmonics $k \Omega$ with $k>1$ can only be suitably expanded, when using at least two shape functions in the Ritz ansatz (1), while most publications restrict to a single one! 
Finally, the amplitude dependence shall be discussed. Fig. 14 shows $\hat{a}_{j 1}$ forming shape $\hat{w}_{3}(x)$ in the case of the intrawell (left) and the interwell solution (right) in the case of different excitation and, therefore, response amplitudes as well. The shapes are almost proportional and are represented for all excitation amplitudes in good approximation by $\phi_{1}$. This changes for the higher harmonic shapes. In Fig. 15 and 16 the shapes for twice the excitation frequency in the case of the intrawell and three times excitation frequency in the case of the interwell solution are displayed. It can be seen especially in Fig. 15 that the corresponding shapes change with excitation amplitude, but all shapes can be very well approximated by $\phi_{1}$ and $\phi_{2}$.

From this it follows that the ansatz (1), (2) with $W_{1,2}=\phi_{1,2}$ can represent all the observed behavior in good approximation, but at least two ansatz functions are necessary while there is no need for amplitude dependent (nonlinear) ansatz functions in that case.

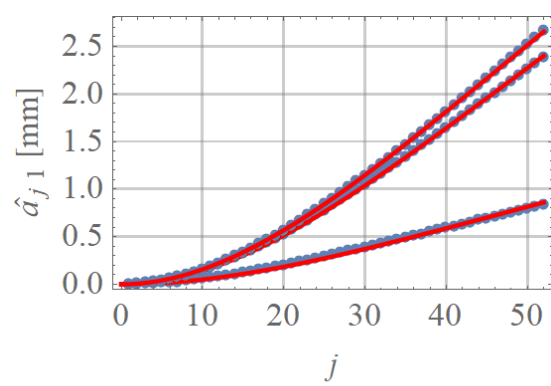

- experimental $-\hat{w}_{1}=\hat{A}_{11} \phi_{1}$

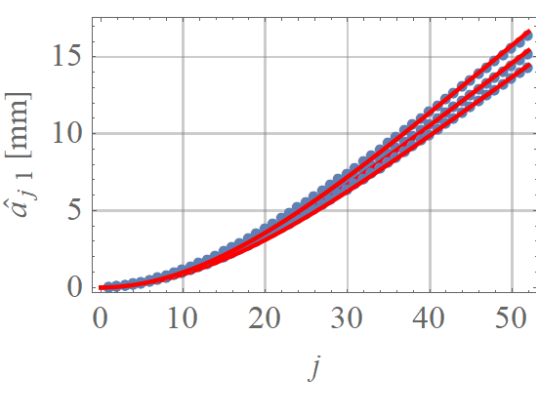

- experimental $-\hat{w}_{1}=\hat{A}_{11} \phi_{1}$

Fig. 14 Vibration shape for frequency $f=f_{0}$ for different amplitudes of the excitation (intrawell left, interwell right)
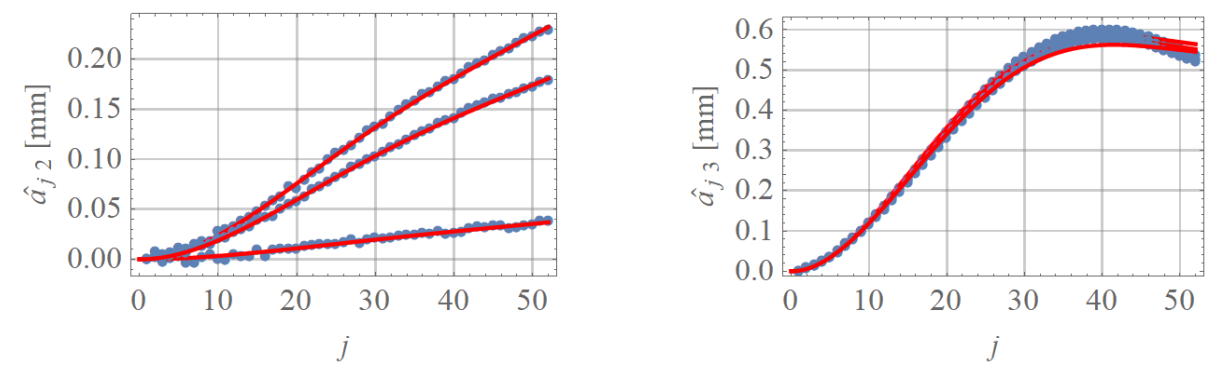

- experimental $-\hat{w}_{2}=\hat{A}_{12} \phi_{1}+\hat{A}_{22} \phi_{2}$

- experimental - $\hat{w}_{3}=\hat{A}_{13} \phi_{1}+\hat{A}_{23} \phi_{2}$

Fig. 15 Vibration shape for frequency $f=2 f_{0}$ Fig. 16 Vibration shape for frequency $f=3 f_{0}$ (intrawell) for different amplitudes of the excitation (interwell) for different amplitudes of the excitation 


\section{CONCLUSIONS}

Energy harvesting performed by a bistable cantilever beam has attracted much attention. In general, the beam is in corresponding modeling discretized by the first eigenshape of the linear beam in a corresponding mixed Ritz ansatz. In this paper, this common assumption has been proofed for suitability. Therefore, the beam has been harmonically excited and corresponding response vibrations have been captured by a high-speed camera. Distinct markers have been applied to the beam and their positions were tracked over a series of frames. In accordance with the Ritz ansatz in theory the experimental results have been analyzed for both intrawell and interwell solution performing the following steps: first a Fourier expansion of the responses has been performed. As there was no period multiplication only the excitation frequency and superharmonics exist in the responses. For these harmonics, corresponding shapes could be identified which are afterwards expanded in the two first eigenfunctions of the undeflected cantilever beam.

The results show that the general ansatz to separate the solution of the beam vibration into a product of functions depending on $t$ and $x$ respectively is possible and sufficient. On the other hand, a good approximation of the experimentally observed shapes can only be reached if at least two ansatz functions are applied. Only for considering the excitation frequency in the response a single ansatz function is sufficient while for superharmonics a second ansatz function is needed to sufficiently approximate the observed vibration shapes. Further, the existing amplitude-dependence of the shapes due to the nonlinearities, can also be covered by the two ansatz functions.

Acknowledgements: This work has been funded by Deutsche Forschungsgemeinschaft (DFG) by grant WA $1427 / 23,2$.

\section{REFERENCES}

1. Priya, S., 2007, Advances in energy harvesting using low profile piezoelectric transducers, Journal of Electroceramics, 19, pp. 165-182.

2. Erturk, A., Inman, D., 2009, A piezomagnetoelastic structure for broadband vibration energy harvesting, Applied Physics letters, 94, 254102.

3. Noll, M.-U., 2018, Energy harvesting system, doi:10.6084/m9.figshare.7492208.v1.

4. Mann, B. P., Owens, B., 2010, Investigations of a nonlinear energy harvester with a bistable potential well, Journal of Sound and Vibration, 329(9), pp. 1215-1226.

5. Harne, R., Wang, K., 2013, A review of the recent research on vibration energy harvesting via bistable systems, Smart Materials and Structures, 22, 023001.

6. Zhou, S., Cao, J., Inman, D. J., Lin, J., Li, D., 2016, Harmonic balance analysis of nonlinear tristable energy harvesters for performance enhancement, Journal of Sound and Vibration, 373, pp. 223-235.

7. Moon, F. C., Holmes, P. J., 1979, A magnetoelastic strange attractor, Journal of Sound and Vibration, 65(2), pp. 275-296.

8. Erturk A., Inman D., 2011, Broadband piezoelectric power generation on high-energy orbits of the bistable Duffing oscillator with electromechanical coupling, Journal of Sound and Vibration, 330(10), pp. 2339-2353.

9. De Paula, A., Inman, D., Savi, M., 2015, Energy harvesting in a nonlinear piezomagnetoelastic beam subjected to random excitation, Mechanical Systems and Signal Processing, 54-55, pp. 405-416.

10. Liu, W., Farmosa, F., Badel, A., Hu, G., 2017, A simplified lumped model for the optimization of postbuckled beam architecture wideband generator, Journal of Sound and Vibration, 409, pp. 165-179.

11. Lentz, L., Nguyen, H. T., von Wagner, U., 2017, Energy harvesting from bistable systems under random excitation, Machine Dynamics Research, 41(1), pp. 5-16. 
12. Lentz, L., 2018, On the modelling and analysis of a bistable energy-harvesting system, PhD Thesis, TUBerlin, Germany, $130 \mathrm{p}$.

13. Shaw, S., Pierre, C., 1994, Normal modes of vibration for non-linear continuous systems, Journal of Sound and Vibration, 169(3), pp. 319-347.

14. Szemplinska-Stupnicka, W., 1983, Non-linear normal modes and the generalized Ritz method in the problems of vibrations of non-linear elastic continuous systems, International Journal of Non-Linear Mechanics, 18(2), pp. 149-165.

15. Mikhlin, Y., Avramov, K., 2011, Nonlinear normal modes for vibrating mechanical systems, review of theoretical developments, Applied Mechanics Reviews, 63(6), 060802.

16. Cazottes, P., Fernandes, A., Pouget, J., Hafez, M., 2009, Bistable buckled beam: modeling of actuating force and experimental validations, Journal of Mechanical Design, 131(10), 101001

17. Nayfeh, A. H., Emam, S. A., 2008, Exact solution and stability of postbuckling configurations of beams, Nonlinear Dynamics, 54, pp. 395-408.

18. Vocca, H., Cottone, F., Neri, I., Gammaitoni, L., 2013, A comparison between nonlinear cantilever and buckled beam for energy harvesting, Eur. Phys. J. Special Topics, 222(7), pp. 1699-1705.

19. Noll,M.-U., 2019, Experimental setup of an energy harvesting system II, doi:10.6084/m9.figshare.7764851.v1. 